\title{
Advanced photonic and electronic systems WILGA 2016
}

\author{
Ryszard S. Romaniuk
}

\begin{abstract}
Young Researchers Symposium WILGA on Photonics Applications and Web Engineering has been organized since 1998, two times a year. Subject area of the Wilga Symposium are advanced photonic and electronic systems in all aspects: theoretical, design and application, hardware and software, academic, scientific, research, development, commissioning and industrial, but also educational and development of research and technical staff. Each year, during the international Spring edition, the Wilga Symposium is attended by a few hundred young researchers, graduated M.Sc. students, Ph.D. students, young doctors, young research workers from the $R \& D$ institutions, universities, innovative firms, etc. Wilga, gathering through years the organization experience, has turned out to be a perfect relevant information exchange platform between young researchers from Poland with participation of international guests, all active in the research areas of electron and photon technologies, electronics, photonics, telecommunications, automation, robotics and information technology, but also technical physics. The paper summarizes the achievements of the $38^{\text {th }}$ Spring Edition of 2016 WILGA Symposium, organized in Wilga Village Resort owned by Warsaw University of technology.
\end{abstract}

Keywords-photonic systems, electron technology, material engineering, electronics, photonics, optoelectronics, lasers, telecommunications, informatics, electronic systems, large research experiments, young researchers meetings, WILGA Symposium, Wilga

\section{INTRODUCTION}

$\mathbf{N}$ EXT year, the Symposium of Young Scientists WLGA Photonics Applications and Web Engineering will mark a modest, yet round, $40^{\text {th }}$ edition and $20^{\text {th }}$ anniversary of its diligent service to the photonics and electronics research communities [1-3]. However, today we discuss a vivid and colourful edition of the WILGA $38^{\text {th }}$, which gathered as every year, for the last several years, more than 350 participants, which included primarily graduate M.Sc. students, Ph.D. candidates, and young scientists, most of them just turned Ph.D., from all over the country and from abroad. During WILGA2016 there were presented more than 250 research and technical works. During the last twenty years of WILGA Symposium activities the aggregate number of participants mounted to several thousands of young scientists. Among them, a large part of them were Ph.D. and graduate students. Thus, today among the electronic and photonics engineers, telecommunications engineers and IT engineers, working at home and abroad, the Wilga has at least several hundred "graduates", i.e. having Wilga in their CVs, with a doctorate degree. This is a significant achievement, indeed. In these

R.S.Romaniuk is with the Institute of Electronic Systems, Warsaw University of Technology. e-mail: R.Romaniuk@ise.pw.edu.pl years Wilga also has published more than 1500 articles indexed in bibliographic and publication databases like Web of Science, Scopus, SPIE Digital Library and others. This considerable achievement is completely unmatched by any other similar conference on electronics, photonics, telecommunications and IT, for young scholars in Poland, and this geographical region [4-16]. The materials of the Symposium have been published in a series of Proc. SPIE for the last fifteen years [spie.org].

\section{TOPICAL SESSIONS OF WILGA 2016}

Wilga Symposium is organized by young scientists from the Institute of Electronic Systems (ISE), Faculty of Electronics and Information Technology, Warsaw University of Technology (WUT) for themselves and their colleagues, young scholars and engineers from Poland and from abroad. The Symposium traditionally uses a few very simple rules of organization, yet not frequently met in other meetings. There is no deadline at all for work submissions before the Symposium. The work results for presentation are accepted until the last day, and the last session of the meeting. Presentations of young researchers, in particular predicted for the publication, should be recommended by their tutors, mentors, or other experienced, senior work supervisors. During each presentation by the young scholar or engineer, there should be a discussion on the essence of the presented study, with the active involvement of the participants in particular topical session. The interest expressed in the presented work during the session discussion may be a factor determining its publication. Unfortunately, a complete disregard of the work, during the session, means frequently no publication for the young researcher. The symposium runs only with single plenary sessions, there are no parallel sessions. The consequence is that it takes up to 10 days to present all papers during the Symposium, as a result of a significant interest of young researchers. Many of the actions undertaken during the Symposium are held on a simple principle of self-organization. The Symposium Sessions are almost always organized by the participants themselves. The participants organize themselves, usually ad hoc in larger or slightly smaller topical activity groups, originating from individual national academic, research and technical institutions or centres. This tradition has developed during the past several years, and is the core of the Symposium today. It involves reporting by the group the currently carried out research topics in their home research centre and organizing around these subjects particular devoted session. The session usually begins with a bigger and more general review plenary presentation on the work of the group, followed by several contributed papers to illustrate the 
practical progress of scientific and technical achievement done during the last year by the group.

Every year the Symposium sessions are slightly different, evolving as a cycle of several years. Some sessions are quite permanent and tied in some way to the Wilga Symposium. These traditional topics are cultivated by both the Symposium organizers - PERG-ELHEP ISE WEiTI PW Research Team, but also by some research groups constantly participating in the Symposium for many years. Some of these more permanent sessions are, for example: Applications of Photonics, Fibre Optics, Sensors, Electronic Systems for High Energy Physics Experiments, Construction of Instruments for Astronomy, Micro-satellites built by students, Free Electron Lasers, Diagnostics of Hot Plasma, Computational Intelligence, Biophotonics and Optogentics, Industry Standards ATCA / MTCA, Precision Time Protocol and Ultra-Precision Synchronization Systems, and some others. This set of very current and interesting topics slightly evolves annually and dynamic young research teams bring new attractive themes, e.g. related to the autonomous moving objects - underwater, ground and flying. The following concise descriptions briefly explain some of the thematic session of Wilga2016 Symposium.

\section{OPTICAL FIBER TECHNOLOGY}

Fibre Optics Laboratory at ISE PW specializes in the technology of fibre Bragg gratings. A FBG is a fragment of the length of optical fibre whose core profile is made refractively periodic along its $\mathrm{z}$ axis. The period of the refractive disturbance is more or less commensurate with the length of the transmitted wave in the optical fibre. Bragg reflection from a number of periodic disturbances creates from a section of the FBG optical fibre a strongly reflective and spectrally narrow, band blocking filter. The FBG is written in a hydrogen sensitized, germanium doped optical fibre, using UV light and diffraction phase mask, or alternatively the Talbot interferometer. After writing by the laser, the grating is annealed in order to consolidate and stabilize its parameters. The method of the phase mask is easy to implement and gives a high repeatability and accuracy of the results. Changing the wavelength of the Bragg diffraction element requires phase mask replacement, which is expensive. The interferometric method is tuneable in wavelength and can write different gratings for various optical spectrum bands. Talbot interferometer containing a set of rotating mirrors is complicated and prone to distortion - there are problems with the repeatability and accuracy of the manufactured gratings. The purpose of upgrading the processing FBG station at ISE PW is to obtain a possibility of producing FBG over a wide spectral range of the transparency of various kinds of optical fibres, to improve the accuracy and repeatability of manufacturing, to provide scalability of the technological automated positioning system based on a single parameter defined by the user - which is the Bragg wavelength for particular fibre type, and flexible/friendly interaction ability with technological apparatus through a full user interface. A development and implementation of algorithm for ultra-precise control of the rotary mirror, and other components of the adjustment of the optical interferometer, has finally resulted in considerable precision and reproducibility increase of the interferometric method, to the level equivalent to that of phase mask method. The parameters of FGBs produced in the ISE PW laboratory are better than the commercial. Work continues on optimizing the technology of tailored FGBs.

In order to produce homogeneous and/or shaped FBG, there is developed in ISE PW a modified phase mask method with a precision nano-positioning. Transmission spectrum of a homogeneous grating (same refractive fringes) contains a welldefined and very narrow back reflection peak. The spectrum includes also a number of reflective, periodic, slowly descending side lobes around the strongest central reflectance peak. Apodized grating has a continuously variable periodic refractive fringe set with a maximum in the middle of its length and fading at the beginning and the end. The refraction of fringes is shaped by a particular monotonous, Gaussian or sinusoidal function, instead of a step function. By continuously changing the refractive periodic perturbation, which is obtainable by changing the intensity of the writing UV radiation during fibre exposure, the reflectance of side lobes in the spectral characteristic of the final grating are significantly reduced. The apodization process, in addition to a significant reduction in side lobe characteristics by at least $20 \mathrm{~dB}$ if not more when optimized, leads also to shortening of the effective length of the grating, and the undesirable widening of the central reflection peak in the FBG spectral characteristic. Modification of the phase mask method consists in introducing an additional degree of rapid, repeatable and easily customizable nano-positioning, which gives the ability to produce gratings of different lengths and variable envelopes of the apodization window.

In practice, during the manufacturing of the grating by using a uniform phase mask, i.e. of constant frequency, there is created dynamically between local mirrors a Fabry-Perot resonator (FP), which leads to an asymmetrical distortion of the spectral characteristic. The grating manufactured in such a way is not fully apodized. The spectral characteristics have side lobes only on one side of asymmetrically distorted main reflection peak. By means of a non-uniform phase mask, e.g. with linearly varying frequency along the mask, a grating is formed for which there are no local FP resonances. The reflection characteristics of the not fully apodized grating is much wider for the main peak and the side reflection lobes are small. Generation of a number of local FP resonances leads to the construction of gratings with shaped spectral characteristics. If spectra of several sections overlap partially then the grating reflection characteristic is very wide, but the band is divided into sub-bands, i.e. within a band there are periodic corrugations of a few $\mathrm{dB}$ to $10 \mathrm{~dB}$. If these sub-bands were divided into deeper valleys e.g. above $20 \mathrm{~dB}$, or more, then a single grating would be multi-narrow-band, or WDM compatible. The aim is to obtain a single filter for the DWDM technology, using a grating optimized in this way. The optimization parameters of the fabrication process are: phase mask chirp, the depth of modulation of the refractive index, and the distance between the maxima of the induced refractive index changes. Usage of intentional scattering techniques, in a form of dithering with respect to the homogeneous mask, when writing the grating, leads to apodization. Longitudinal nanovibration of the phase mask gives the possibility of additional control and adjustment of the visibility of interference pattern 
formed on the photosensitive core of the fibre. The interference pattern is blurred until it disappears in the case of perfect phasing of the dithering with period of the grating. For small values of the dithering, achieved with a nano-positioning stage, constant value of effective refraction is obtained and the local FP resonances are not present. The shape of the refractive envelope function, called apodization window is triangular, but can also be other function like Gaussian, $\cos ^{2}$, depending on the nature of the dithering function. Optimized dithering of the uniform phase mask leads to writing the apodized grating with reduced side reflection lobes by more than $15 \mathrm{~dB}$ in the spectral characteristic.

There were studied the characteristics of FBG in the ionization field environment. Irradiating by neutrons and gamma rays in the range of tens and hundreds of $\mathrm{kGy}$ shifts the Bragg peak of the component towards longer wavelengths. The magnitude of the displacement depends on the type of the fibre material (glass, dopant, polymer), and the method for writing the grating. The Bragg wavelength shift equals from single pm (polymer fibres) up to tens and hundreds of picometers in various kinds of glass optical fibres.

FBGs are used for the construction of multi-sensors. Examples of such components include the simultaneous measurement of strain and temperature, liquid level and temperature, and others. Bragg gratings of different sections with different characteristics may be combined in a single sensor. Skew gratings (with a small angle of inclination to fibre axis, of the order of single degrees) have typical spectral characteristics like in standard devices, but additionally for shorter wavelengths they have a lot of bands associated with cladding mode resonances. The position of the major spectral reflective band depends on the inclination of the grating and the wavelength of the cladding mode resonances depends on the sum of the effective refraction of the core and eigenrefraction of the individual cladding modes. Sensing properties of the inclined grating are thereby modified. Cladding mode resonances are sensitive to the environmental refraction which can be used for biochemical sensing. The core resonance is sensitive to fibre stress which is used to measure mechanical properties, like strain, vibrations, bending, etc. A combination of both sensitivities can be used to measure the temperature and thermal properties of materials. The differential sensitivity is of importance in such multivariable sensors. Changes in temperature shift spectrum as a whole, of the order of several-several tens $\mathrm{pm} /{ }^{\circ} \mathrm{C}$. Change of the immersion depth of the sensor reduces only the level of optical power carried in cladding modes across the spectrum. A prototype of the FBG sensor has been built and the tests were performed in a thermal chamber with different liquids. For paraffin and a particular FBG device, the obtained sensitivity was linear with immersion, and was equal to approx. $0,5 \mathrm{~dB} / \mathrm{mm}$. The device had also a small thermal sensitivity of the optical field of cladding modes equal to $-0.1 / \mathrm{mm}$. The aim of the research on these sensors is that they can be used in the chemical industry, food production, and in harmful conditions.

FBG are written on an optical fibre taper to shape the spectral characteristics, reaction sensitivities and optimize the group delay and phase characteristics. Variable parameters are the shape of the tapered transition part of fibre and apodization window. Modelling of conical FBG may be performed by full analytical method or by differential method, dividing the grating to uniform lengths with different geometrical parameters from the neighbours - the diameter of the fibre and optionally also the grating period. A uniform grating is relatively easily modelled using a matrix notation. The modelling takes into account the physical characteristics of the fibre taper, and in that the dependence of effective refraction on fibre diameter, modal field propagated in the core with variable diameter of the cone. Modelled characteristics of the conical grating, taking into account boundary conditions, were compared with the measured ones performed on fabricated components. Requirements imposed by the optical fibre telecommunications system relate to the group delay linearity and lack of its oscillation. Hence stem the requirements for the formation of the transitional area of fibre and apodization window. Initially, a linear cone was assumed and apodization window as the tanh function. In the case of an apodized grating we are dealing with a constant effective refraction and in the case of a not fully apodized one, the effective refraction depends on the apodization profile. Minimizing the oscillation of group delay is associated with a reduction in the FabryPerot resonances.

\section{HEP EXPERIMENTS, COSMIC RADIATION, TOKAMAKS}

High-energy physics experiments are related to elementary particles, interaction of heavy ions, study the properties of compressed baryonic matter, etc. Work on such experiments, in terms of instrumentation development are carried out at WUT, Faculty of Physics and ISE. The LHC detectors investigate mainly $\mathrm{p}-\mathrm{p}$ collisions, but also $\mathrm{p}-\mathrm{Pb}$ and $\mathrm{Pb}-\mathrm{Pb}$. The ion collisions are also investigated by the CBM experiment at FAIR in Darmstadt. Strongly interacting matter created in the collisions of heavy ions is described by the language of thermodynamics using such parameters as temperature, phase transitions, chemical potential, etc. The resulting "ball of fire" is small and of short duration. To determine the characteristics of the changing phases of matter, if only any quark-gluon phase QGP is created, followed by a mixed phase (if not, then we have a hadronic phase), there is needed a sufficiently large volume of new matter and time of observation. The observation period shall be extended by frequent repetition of the fireball generation. For much larger volume of heavy ions collision like $\mathrm{Pb}-\mathrm{Pb}$, where there exist a local structure of QCD vacuum (as in a pp collision), the initial phase of cold nuclear matter (as in $\mathrm{p}-\mathrm{Pb}$ collision) and QCD plasma, a reference system (not fully confirmed) are the $\mathrm{p}-\mathrm{p}$ and $\mathrm{p}-\mathrm{Pb}$ collisions. It is important to discover the details, using the instrumentation in which design and implementation the WUT ISE is involved, of the collision evolution of heavy ions. A sign of the development of QGP phase, or strongly interacting matter, during the collision, is collective movement, hydrodynamic flow, dominated by radial flow and elliptical (triangular) flow as azimuthal modification. The research is focused on thermal emission from the collective medium, whose volume and lifetime increases strongly with the energy of the collision.

The research on phase diagram of strongly interacting matter (SIM) is carried out in several laboratories. The experiment STAR at BNL/RHIC leads the BES project (beam energy scan). The project is currently being upgraded into a BES II having greater opportunities to identify particles. Au$\mathrm{Au}$ ions are collided in the energy range $7.7-200 \mathrm{GeV}$. LHC 
experiments explore the phase diagram space for low baryonic energy but for substantial temperature. A dedicated experiment in the LHC complex is NA61-SHINE at SPS with heavy ions and neutrinos. RHIC has a considerable scope of energy scanning to large values of the baryon chemical potential. However, now it is observing the events too late, after partial kinetic defrosting of particles, i.e. after hadronization. Such a broad energy scanning capabilities allow accurate search for the QCD critical point, transition phase signals or the phase boundary. Earlier observation in time are required. Future experiments in the FAIR operate above the curve of the first phase transition in terms of a significant baryon chemical potential, however, below the range of neutron stars and colour superconductor.

FAIR will have the possibility of early fireball observation still before hadronization. In the SIM phase diagram, at phase transition of the first order, the first derivatives of state parameters are discontinuous, i.e. matter and energy densities, etc. This is similar to the case of phase transition of water and ice. This means that a smooth transition between phases of matter occurs with the coexistence of phases within certain parameters region in the vicinity of the critical line and point. The phase transition of the first order demarcates hadron gas from the quark-gluon plasma. In the hadron gas, quarks and gluons they are trapped, and this matter has hadron, meson, and baryon degrees of freedom. During QGP, the quarks and gluons are quasi-free and the matter has parton degrees of freedom. The critical point is localized in the temperature of $170 \mathrm{MeV}$ and a relatively small baryonic potential of the order of $300 \mathrm{MeV}$.

Higgs boson, and its parameters - CP, spin, coupling constants to fermions, are under further research. The SM assumes the spin and CP of the Higgs boson to be $0+$. To determine the properties of the particle, much more data is needed. Decay channels of $\mathrm{H}$ with probabilities from $60 \%$ to $0,01 \%$ are: bb, WW, gg, $\tau \tau, \mathrm{cc}, \mathrm{ZZ}, \gamma \gamma, \mathrm{Z} \gamma, \mu \mu$. With today's technology it will take many years of experiments to gather relevant amount of data. The key to increasing the effectiveness of research is the modernization of equipment and software in subsequent periods of operating the LHC accelerator complex. In the CMS detector, during the LS1, there were modernized many elements and system components including the exchange of the whole new first level trigger FLT (L1) for the detector overlapping areas. Extremely demanding triggering system contains 75 million readout channels, running at $25 \mathrm{~ns}$ clock and data rate of TB. The primary data stream is processed on-line and approximately 100 events are extracted for further off-line analysis. The FLT is designed as a dedicated electronics and HLT as software on a computer farm. The injection frequency of input data is up to $100 \mathrm{kHz}$. The FLT operates with the algorithm for finding the trajectory of overlapping muons (OMTF). The FLT program components are SWATCH, and a common, standardized control applications environment and a GUI; microHAL (uHal); high level equipment control library; which is used by all the new hardware modules of CMS. OMTF environment consists of the operating system and equipment control. Algorithms and firmware are tested using cosmic rays. The new generation system trigger replaces the previous CMS L1 trigger.
A session devoted to tokamaks was organized by the collaborating research groups from IFPiLM and ISE PW. Research on tokamaks is associated with plasma fusion and thermonuclear energy generation. The fusion of light ions requires overcoming the Coulomb potential barrier. The critical temperature to overcome this barrier is of the order of hundreds of millions K. High probability particle tunnelling phenomena are used instead, to overcome the need for excessive temperature. The fusion reaction provides a large amount of energy because is associated with a deficit of mass. This reaction occurs in the Sun and the stars, but the conditions for such a reaction are not directly realizable on the Earth. It is necessary to use other reactions like DT $(17,6 \mathrm{MeV}), \mathrm{DD}$ (3 to $4 \mathrm{MeV})$, DHe (18.3 MeV). Trapping of a stable, high temperature, circular plasma jet is done in a donut shaped tokamak vacuum chamber. The plasma current of poloidaltoroidal-helical magnetic field is a secondary circuit of the transformer. The plasma is heated and maintained by a variety of methods using toroidal coils and high intensity RF fields. The experimental tokamak, where the works are carried out, is JET in Culham, and the future target machine is ITER. To initiate the fusion reaction the following threshold conditions must be satisfied: ion plasma density, plasma temperature, and reaction time. As a result of interaction with the chamber of the tokamak the hot plasma becomes contaminated. It is necessary to carry in real time the tomographic observations of plasma. Hot plasma tomography development was presented during one of the dedicated sessions in Wilga.

The electronic test equipment for plasma diagnostics in JET tokamak, evolves in the direction of remote automation configuration. The necessity of automatic configuration results from an increasing complexity and large number of integrated circuits that support the system, and first of all the increasing complexity of undertaken experiments. The research is conducted in collaboration between ISE PW and IFPiLM. The construction of the plasma tomography detector is as follows. Outside the radiation window defining the sensor aperture there is triple stage, gas chamber, pixel electrode GEM detector, followed by a readout PCB. The pixel signals are fed to the analogue input stages of the readout board, behind which is a high voltage power section $5.5 \mathrm{kV}$ for GEM detector. Multi-channel signal from GEM detectors is input to multiple 64 channel acquisition sections, and then to the FPGA backplane boards. The analogue input boards, resistant to ionizing radiation, are connected to the 16 channel ADC boards and then to digital backplane boards. The PC environment is connected to the router and PCIE interface and USB configuration link. The PC environment does data processing and manages the measurement system. Developed firmware supports a new version of the hardware. The firmware includes support for: data transmission channels, diagnostics, control and registers status, DMA, memory status, Wishbone bridge, and PCIE-G2 2.5 Gbps. The system has the ability to detect configuration. The automatic configuration is based on a standard FCS environment. The environment includes drivers for boards, integrated circuits, and communication interfaces. FCS allows for monitoring of the main power supply, thermal protection, clocks, amplifiers, signal levels of voltage and current, and boards positions. The system integration was done by developing a common 
software interface for internal and external software. The system shall be further developed to increase the level of integration, in particular including the full support of analogue components, the integration of MatLab scripting, etc.

One of the directions of development of the software and hardware diagnostic of hot plasma is the integration of a number of platforms, including optimization of Matlab script in order to obtain the maximum throughput in the processing of the large data stream. Other tasks include increasing the precision of the separation of overlapping pulses from multipixel (currently 256) detectors GEM, as well as the construction of stand-alone software module for a flexible and scalable, next generation diagnostics system. Works on the development of algorithms and their optimization for hightemperature plasma diagnostics are run in ISE PW. The system is implemented in the WEST test project. The Mex technology used for serial DAQ is accelerating the calculations almost by two orders of magnitude, due to efficient parallelization. The performance effectiveness is compared with FPGA chips. There are implemented additional diagnostic structures for the construction of histograms and extended statistics of the events. The work progresses on the problem of variable-length data sets.

A research group of IFPiLM is involved in the construction of instrumentation for the Wandelstein stellarator W7-X. W7$\mathrm{X}$ is an experimental fusion reactor designed for continuous operation, based on the multi-period Helias configuration. The magnetic field is generated in a single coil configuration with no longitudinal current in the plasma and hence without a transformer. The first measurement campaign was held in the period December 2015 - March 2016. There were available around 20 plasma diagnostic systems, including a number coauthored by several national teams. Plasma 2D tomography is performed using GEM detectors. The quality of the heated plasma is evaluated. PHA circuit for measuring impulse heights was tested. The purpose of this measurement system is the identification of medium and high impurity levels in plasma, i.e. atoms having large $\mathrm{Z}$ number, and to determine electron temperature from the slope of the energy continuum curve. Plasma is observed by the PHA system close to the canter of the stream. The detectors used large-surface polycrystalline silicon sensors covered by $\mathrm{Be} / \mathrm{Al}$ window. The range of measured photon energy is $250 \mathrm{eV}-20 \mathrm{keV}$. The detectors cooperate with preamplifiers placed outside the vacuum chamber. PHA system components are as follows: SDD detector, preamplifier, multi-channel digital converters, USB-optical fibre converter, fast transmission line, and finally PC environment. The PHA system was installed on the W7-X before the first measurement campaign. The main impurities have been identified as $\mathrm{S}, \mathrm{Cl}, \mathrm{Ar}$, and Ne. Plasma temperature and pollution are subject to precise estimation.

The WEST project is testing, in the long-pulse tokamak Tore Supra, the implementation of actively cooled tungsten divertor, similar to that planned for the final solution of ITER machine. In metal tokamaks the interaction between the particles transport and magneto hydrodynamic phenomena leads to the accumulation of impurities and finally to the operation interruption of the whole machine. The aim of the research is to obtain a stable plasma discharge of as small interaction with the walls as possible. The studies require the use of new diagnostic tools of hot plasma stream in the whole cross section. This tool is a poloidal diagnostic tomography focused on metal pollution, radiation of these pollutants, and especially on tungsten. The requirements for tomographic image are as follows, and relate to the spatial and temporal resolution and energy discrimination: spatial resolution better than $1 \mathrm{~cm}$, time resolution better than $1 \mathrm{~ms}$, energy discrimination in five predefined spectral bands. There was used SXR tomographic diagnostic system. There is developed a new poloidal tomography system using GEM detectors. The measuring system must be resistant to many types of radiation which source is the fusion reactor. This is in particular the neutron radiation which may lead to activation of the material and distortion of the detector measurements. In order to determine the potential level of GEM detectors activation, they were exposed to radiation from the Am-Be source. A greater impact on the detector performance was observed from highenergy photons than from neutrons. The first tests were performed in the modernized ASDEX infrastructure.

Measurements of cosmic rays are performed by a number of experiments, including PAO (Pierre Auger Observatory) having $3000 \mathrm{~km}^{2}$ area in Argentina. Faculty of Physics, University of Łódź is involved in this experiment. The high energy cosmic rays observed on Earth have a number of sources. Not all have yet been confirmed. Not all acceleration mechanisms of cosmic ray particles are recognized. The direction of arriving cosmic radiation is altered by magnetic fields in the interstellar space. A very small stream of particles is observed for the most energetic radiation. On the spectral curve of cosmic radiation there are characteristic points called bends - knee $10^{16} \mathrm{eV} \mathrm{m} / \mathrm{s}$ and elbow $10^{19} \mathrm{eV} \mathrm{km}^{2} / \mathrm{y}$. Particle flux change spans over 32 orders of magnitude inside the energy range $10^{9}-10^{21} \mathrm{eV}$. For smaller energy than $10^{10} \mathrm{eV}$ the stream becomes saturated at $10^{4}\left(\mathrm{~m}^{2} \mathrm{sr} \mathrm{s} \mathrm{GeV}\right)^{-1}$. Cosmic rays reaching the Earth's surface are initiated in the upper atmosphere by protons and nuclei, up to iron. In the radiation reaching Earth there are three components - hadron, muon and electromagnetic. The PAO detectors consist of 1680 Cherenkov sensors and 4 fluorescent and radio receivers. The detector electronics is modified in the direction of noise reduction and detection sensitivity increase.

With the fast development of the available computational and memory resources in electronic systems, there is changing completely the approach to their operational software. The extensions are not only for the basic functionalities but as well for intelligence, additional diagnostics useful for the operator, graphical interfaces for particular serviced experiments, and all kinds of visualizations of data, events, apparatus state, transmission network, software, multi-layered monitoring system, including multi-channel monitoring of equipment as well as data quality monitoring. Each of the experiments generally performs its own visualization software. Although in this regard, standardization is progressing as part of larger software development initiative and functional programming environments like VML.

The development of visualization techniques of the physical events are shown here on the example of ALICE/LHC experiment. Visualization of the events helps considerably the operator to work with the complex networks of detectors. Visualization features that designers use for different parts of 
the system are, for example, positioning of hits for geometry testing, diagnostics system - understanding and prevention of subtle problems, events overview and their classification for the analysis, search for noise sources, cross-validation tests for event reconstruction programs, analysis of the cosmic background, illustration of angular relationships between particle tracks, visual inspection of various types of algorithms for noise reduction, event reconstruction and adjustment, display events for physical analysis and for education, production of documentation for archiving purposes, conference presentations, popularization, outreach, etc. There was presented the visualization architecture of Alieve system for the Alice detector. One method to improve the visualization for the operator is the use of multiple monitors to see the 3D effect. Data and physical phenomena visualization is an important direction of development in the construction of a new operator interface for all HEP experiments. The 3D effect can be obtained through the use of equipment like VR Oculus-Rft, as well as via the use of $3 \mathrm{D}$ monitors with passive or active glasses.

Multiple monitor based solution lacks today an interactivity feature with the operator/viewer. The contents displayed on the screen does not move when the user moves his head - there is no feeling by the viewer of looking into the real window. In the case of multiple displays, the contents does not shift mutually between them. Well known for several years and stabilized computer games techniques come of help, like the 3D Surround. This technique supports the user employing multiple monitors, usually three, but sitting motionless in a well-defined place. In the case of a moving user or multiple users the simultaneous 3D Surround may be unstable. Creating an efficient and cost-effective visualization of events and data is very important for HEP experiments in CERN for the accuracy of media content, the convenience of the operators, but also for the purpose of popularization of science. Changing the content displayed on multiple screens requires position tracking of the system operator/observer. Feeling of being surrounded by an observer is enhanced by angular setting of the screens. To build the upgraded visualization system two cameras were applied, arranged separately from the monitors and tracking the position of the viewer. The next step is to develop a global and highly standardized visualization system at a higher level for CERN. This will be the proposed Total Environment Display of events, or TED. It is a global project of CERN Media Lab. It is based on the Unity, an engine of computer games. It is modular and applicable on any platform. It is expected to be used by the whole LHC complex in the close future.

The Pi-of-the-Sky (PI) project is involved in the works of the LSC-Virgo EM Follow-up (of LIGO GW discovery) consortium about looking for transient electromagnetic phenomena partners of gravity wave candidates. Finding such associated EM transient phenomena could be the beginning of a new field of multi-signal, or exactly hetero-signal astronomy, investigating correlations between gravity waves and electromagnetic waves. The measurements campaign of LSCVirgo EM Follow-up was carried out between September 2015 and January 2016. Finding a correlation between gravity and EM wave would be a significant breakthrough and extension of available tools for the experimental astronomy. The PI project, originally designed to observe relatively bright (limited to approx. 12.5 magnitude, exposure time $10 \mathrm{~s}$ ) and short optical transient signals, has two observation stations in INTA, Spain and SPdA in Chile, and previously at Las Campanas. The separation of $8500 \mathrm{~km}$ between stations allows parallax observations. The current PI consortium consists of CTF PAS, CAMK, UW, NCBJ and previously also PW. The current project is a continuation and modernization of the previous one, covering the entire Earth. The modernization included: advanced detectors, Luiza software framework, GLADE catalogue, etc. Current global network of interferometric detectors of gravitational waves includes the LIGO-Hanford, LIGO-Livingston, VIRGO, GEO, and planned LIGO-India and Kagra. The Advanced LIGO provides the increase in the detection range approximately 10 times in 2021r (currently 4 times). The algorithm of observation in the LSC-Virgo Follow-up project is synchronized to central alarms and whole night sky scans - wide or deep, depending on the capabilities of the participating observation stations. A EM partner for GW150914 collapse of two black holes is searched. Another source of gravitational waves may be a supernova star. The PI project develops observation algorithms for relevant supernova stars. The algorithm has an observational asymmetry due to the relatively greater ease of observation of new objects present in the watched scene, than faint objects disappearing from the scene. For objects disappearing there are used quite different tools. The lost object is of interest only after a few nights. In addition, the sensitivity of PI observations is not the same from night to night. The limit magnitude is variable. Some variable stars are dimmed below the value of limiting magnitude. Observations are difficult in the galactic plane because of the density of objects. There are also observational interferences originating from passing satellites.

The project PI participates in collaboration Vipers VIMOS which is a public beyond-galactic review of the redshift. The overall objective is to study the large scale structures and cosmological parameters in time half the current age of the universe. There were measured redshifts of 105 old massive and passive galaxies in the range $0.5<\mathrm{z}<1.2$, clusters of galaxies, expansion of the large structures and properties of the galaxies. The observed galaxies have areas of stellar populations passively evolving with no signs of the formation of new stars. These types of galaxies are standard space chronometers, allowing for speculations regarding the potential to go beyond the local universe. Astronomical observation equipment of the PI project is subject to constant modifications. In ISE PW there is build a new version of the advanced astronomical camera, based on Zynq SoC. In order to obtain a low quantization noise there is applied a highresolution ADC. High resolution quantization means, however, significant limitations of the sampling rate. The method of sigma-delta modulation provides several $\mathrm{MHz}$ bandwidth, slower than the readout clock, and SAR methods provides bandwidth up to tens of MHz. It incorporates several SAR ADC circuits with phase shifted sampling clocks. The oversampling of the signal reduces quantization noise. The least significant bit was omitted in order to minimize non-linearity between the devices. ADC 18b, $5 \mathrm{MHz}$ circuit was used. The algorithm for pixel data management was optimized. Asynchronous readout of ADC-FPGA Serdes was applied. 


\section{Sensors, MEAsurement Systems, CyBERSECURITY}

Sensors designed to work in an environment of significant concentrations of methane $\mathrm{CH}_{4}$ must cooperate with spark-safe apparatus. Work on intrinsically safe sensors designed for operation in the coal mine are carried out at the Technical University of Lublin. To support the sensor and other devices such as water pump, cooling system, gas pump, there were used BLDC brushless engines, controlled by optimal PID device.

Microfluidic sensors are tested in the IMiO PW laboratory. In such sensors there are used $\mathrm{NiCr}$ micro-heaters as actuators and functional elements. Microfluidic sensors are implemented in fibre optic systems using micro-capillaries. The head of the sensor consists of two main elements, the base and capillary optrode. Exchangeable optrode is partially filled with the test liquid. The base of the micro-capillary photonic system is used to heat local spot in the optrode and create a gas micro-bubble from the test liquid. Light propagation is monitored in the heated part of the capillary. Micro-bubble generation switches off the heating immediately. The circuit of microfluidic sensors includes: input and output fiber optic, light source and detectors, photonic sensors, capillary circuits, micro-heaters, power supplies, integrated positioning, etc. Thin-film technology (100 nm heating layer and $350 \mathrm{~nm}$ ohmic contacts) of $\mathrm{NiCr}$ micro-heaters on alundum ceramic substrate was developed at the Technical University Lublin. Micro-heaters were comprehensively characterized electrically and thermally, statically and dynamically. The sensors were subject to optimization technology. Anti-oxidation layer of silicon nitride was used to protect the sensors.

Sensors for use in the hardware layer of the Internet of Things are growing rapidly due to recent advances in the integrated and hybrid technologies, including MEMS, MOEMS, and the like. Miniature integrated magnetometers employ the phenomenon of anisotropic magneto-resistance. Magnetometers emit the EM field, as they require periodic magnetization, and one of the optimization problems is to reduce this emission. Miniature magnetometers are used for magnetic measurements, including the detection of deformation of the Earth's magnetic field by the ferromagnetic objects, general detection of objects, vehicles, monitoring integrity of the construction, building security electronic and magnetic detection, direction of movement of objects, object identification, protection of objects and subjects, reduce the level of external visibility in safety equipment, etc. Work on a miniature, integrated quiet magnetometer is conducted at the Technical University in Ostrava. Magnetization is carried out using a non-periodical signal and spread spectrum techniques. The radiation sensor is masked by extending the narrowband noise in the wideband signal, using the pseudo-random codes, of low power density. The sensor is magnetized in an asynchronous manner.

The construction of large area $\mathrm{SiC}$ photodiode based sensors require cooperation with dedicated amplifiers. Work on such systems are carried out in IMiO PW. Large photodiodes are used in the construction of optoelectronic sensors working in harsh environmental conditions, including the detection of UV and X-rays. Working in the adverse environment, the sensors must demonstrate resistance to the harmful effects. Mitigation of the interference is performed at the hardware level, signal, or programming levels. Immunization of the sensor to constant and low frequency signals, is based by the additional modulation of the transmitted light by and additional useful signal, which can be filtered out precisely by the measuring system. Large area diodes have significant capacity which is variable with polarization. This capacity modifies the conditions of element cooperation with the sensor preamplifier. In order to optimize the preamplifier there were analysed theoretically by simulation and practically two circuit geometries: a trans-impedance amplifier and the inverting voltage amplifier with negative feedback. Both eliminate feedback DC signal. Both types of preamplifiers have slightly different characteristics of the application.

In the Perg ISE laboratory there are built a lot of practical sensor systems. Non-contact thermal sensor are used for traffic control, presence of lighting, movement of objects, intrusion detection, etc. For the construction of the device there were used the most popular industry standards like: cheap microcontrollers, UART, I2C, and Bluetooth 4.0 I/O. The device has the ability to remotely adjust the lighting, measures the level of external lighting, detects motion, is also a sensor of smoke and fire.

Lublin University of Technology, EE Faculty is working on small, kilogram size, autonomous and remotely controlled drones, equipped with numerous sensors. Practical and experimental uses of such devices are getting wider. They are divided into a growing number of sub-types - micro-planes, helicopters, flying wing, etc. The used drivers are electric brushless BLDC motors, engines with internal combustion for liquid fuel and gas. A test bench was built to simulate the flight environment and for evaluation of micro-motors of the order of a single $\mathrm{kW}$ for gas and petrol. There was built a flying model with a test engine in order to verify the device performance. The engine was characterized in terms of the usefulness to the drone model. In particular, the inertia of the combustion engine were rated in comparison with an electric motor. To identify the system under test and evaluation dynamics, the ARX method was used. One of the research purposes is a precise control and optimization of fuel consumption of the drone.

In several academic laboratories, including the AGH, PW, Lublin University of Technology and other ones, the scientific and technical works are carried out related to various aspects of the construction, optimization, testing and use of generally local photovoltaic systems. The test photovoltaic systems are equipped in many sensors allowing to assess the state of the system operating conditions, the reliability of new solutions, efficiency and its changes over time and different operating conditions. Laboratories working on new technological solutions of photovoltaic cells are at AGH, IFPAN, PW and other academic and research labs. Many of the small and medium-sized experimental systems are tested beyond the power grid. Experimental panel, or set of panels, is connected through a regulator with a battery and DC/AC inverter. The inverter regulates the load through the filter and the phase is connected to a nominal standardized network of loads. Photovoltaic power test systems are equipped with an extensive layer of monitoring. Monitoring system is supplied by large amount of data from readout points positioned at critical points in the PV system. There are many commercial solutions of such multi-microprocessor controllers, connected with a multi-channel readouts and on the other side connected 
with a PC through the USB link. The controllers also are built into the proprietary solutions with their own software. The controller in the operator panel shows the current status of the source or network of sources, shows signal waveforms in real time, performs data acquisition, calculates adequate statistics, has built-in alarms, etc.

Construction of flexible measurement, diagnostic and advanced functional systems that support multiple peripherals and sensors and are processing large amounts of data, requires currently high efficiency of programming the multiple PLC/FPGA circuits. Commercial CAD systems and programming environment such as Quartus, Vivado, and ISE are frequently not optimal. They are based on a heuristic approach that works well because of the considerable and redundant resources currently available in the FPGA. The classic approach, using the methods of rigorous logic synthesis, leads to optimal usage of the resources. But not everyone is interested, if your set, not extremely demanding, functionality works properly. With the approach of heuristic optimization the problem is not resolved, only smoothly working required function is needed. The use of large memory resources can be effectively optimized using formal logic synthesis methods. Such works are carried out in cooperation of ITele and ISE PW. Memory based implementation solutions require consideration of two fundamental issues. It is necessary to reduce the number of variables that determine the function. Large function is decomposed into a network of smaller subfunctions in a way that maintained the properties of the original function. The developed algorithms reduce the number of variables using the matrix of the tags and features. The algorithm uses conventional synthesis procedures to complete Boolean functions. Decomposition of the function is based on a partitioning of input variables so as to obtain a two-level functional relationship.

Small and large devices and functional systems, such as mobile phones, mobile autonomous objects, cars, household appliances, intelligent home environment, and many others are equipped increasingly frequently in smart sensors. Work is underway in ISE PW labs on the construction of an autonomous cleaning robot, recognizing the type of surface to be cleaned. The robot adapts to the recognized surface a relevant set of cleaning tools. The type of surface and the distance of the robot base from the cleaned surface is measured using a set of ultrasonic sensors. Several sensors based on parameters such as directivity, price, resistance to water and dust, mechanical shock, and working in reflection geometry were chosen for tests. The measuring algorithm determined first the type of surface and then measured the distance. Measuring the distance must be scaled by the type of surface reflection. The construction of the transmitter and the receivers must ensure the prevention of direct coupling between them. To identify the type of surface there was used in addition MEMS-based inertial sensor. The current recognition system which was based on a set of several ultrasonic sensors made mistakes recognizing similar but uneven surfaces. In the tested solution the ultrasound system is supported by an accelerometer. The requirement is the full integration of new sensor with the existing system in terms of hardware and programming. The system uses CAN bus. STM32 microprocessor was used and three accelerometers, CAN interfaces, USB and SWD. Robot behaviour was tested on a number of hard and soft surfaces, textured, rough, smooth, and the like. Recognition takes place on the basis of sensors fusion and signal processing. Dry tests were carried out using recorded signals from the database and the developed decision algorithm, containing statistical block. The system is under further developed for the detection of subtle recognition of more different surfaces. In addition to the work on the development of the ability to recognize the surface by the cleaning robot the works are carried out on the development of the internal network architecture. This is due to the continuous complexity of the system, increase functionality, dramatic increase of the number of supported sensors and tools. The robot has among other things, wi-fi, GSM, GPS, sensors, peripherals, support for a ride, cleaning tools, material resources, power, etc.

If the job is bigger, including cleaning large areas, doing more differentiated tasks such as monitoring of limited space, it can be done by a group of cooperating robots. Developed are cooperation algorithms of autonomous robots - a swarm of intelligently interacting machines. The algorithms include among others, such features, attributes, and parameters as: definition of tasks, area of operation, the establishment of a network of communication between members of the group of robots, splitting of tasks between the units at the beginning of the operation, modification of tasks with the progress of work carried out by the group. The communication network is many points to the many points that is, each to each or multicasting. The network is fast reconfigurable after the communication error. Communication and scalable routing can be based on the principle of relay with testing of the transmission quality of the path and the dynamic change of the path in case of poor signal quality. Individual robots have access to all of the group data. The processing of local data is held locally. Robots are "aware" of the task distribution between the operational units. The tasks are modified in the event a nearby region shows the lowest progress of completed tasks. Modification of the task is done on a one to one negotiation between adjacent machines. To test a particular version of an autonomous cleaning robot there were added additional sensors. The sensor detecting the places specially dirty sensitizes the machine into a special mode. The strength of the cleaning by the robot depends on the operation of such a sensor, and the indication of dirty position on the floor. The sensor operates on the basis of image analysis and processing.

One of the ways of integration of sensors are methods based on common technological substrate to build a multifunctional complex systems. There are tested solutions of a whole laboratory-type integrated circuit on a common photonic substrate, or on optical fibre in particular using photonics micro-capillaries, and the like. Research is conducted on laboratory on a thin-film in ISE PW. The sensor can integrate several functions in a single miniature $1 \mathrm{~cm}^{2}$ structure. The aim is to build a simple, low-cost, single-use disposable, thin-film sensor for measuring the concentration of a liquid substance. The film structure has inputs and outputs of the analysed medium, micro-channels and a complex system of parallel and perpendicular electrodes. The tests of a fabricated prototype were performed on a variety of water soluble materials by static measurement of the RLC. A miniature target system was built on the STM32 microprocessor and integrated capacity meter AD7745 using I2C communications. 
In another solution, the thin film sensor used as an active element an optical fibre cone placed in the microfluidic channel serving as sensitive refractometer. Refraction is closely linked functionally with the concentration of the measured substance in the microfluidic channel.

Interest in shale gas made it necessary to develop a system for measuring multiple parameters related to its potential exploitation. Work on the measuring system are carried out in ISE PW. The measured parameters are associated with the gas identification, measurement of gas parameters, measurements of different physical and chemical values, but also the pollution of the groundwater. Measurements can be made deep in the well and near the drill, but also on the surface. The measuring system with sensors, information processing systems, data transmission network is fully programmed at the level of the operator. Work on the measuring system is a part of a large project related to the monitoring of the dangers caused by the exploitation of shale gas and stages of project life from the start, through industrial activities, termination, and then re-cultivation of the exploited land. In the first stage of the project the measuring station is a device which measures online groundwater and atmospheric conditions. The data is formatted in the station and sent to the server. The station equipment is standardized for the industrial environments and includes several types of power systems, industrial computer, interfaces and connectors for sensors, and GSM antenna. The measured parameters of water are as follows: conductivity, temperature, $\mathrm{pH}, \mathrm{Cl}$, salinity, total amount of the dissolved solid material in water, and the level of ground water. There are applied several industrial integrated measuring sets of these parameters. Weather Station is measuring a few typical parameters. The software supports control sensors, telemetry measurements, diagnostics, data archiving, and communication with the server.

A group of sensors of various sizes for mobile applications is designed in the technology compatible with the Internet of Things. The works are conducted in the Microsystems and Sensors ISE PW lab. Such sensors often require labelling of the measured data with the time and GPS parameters. There are constructed different versions of the full tracking systems designed for use with sensors and meet standards of IoT. The device consists of a hardware layer, Internet connectivity, $\mathrm{M} 2 \mathrm{M}$ protocol support and applications - most often operated from your smartphone. The hardware typically includes multiple sensors, a microcontroller, battery and power systems, GPS locator and GSM network connection. If the unit is being built in the stand-alone version, one of the requirements is to optimize energy consumption, both in the data transmission mode and sleep mode. Lublin University of Technology developed monitoring methods for wireless ECG signal and PQRSTU signatures, similarly using a target IoT technology. The system uses multiple sensors, like 3 axis accelerometer, 2axis gyroscope, associated with the monitored person: sampling occurs with a frequency of $500 \mathrm{~Hz}$, measurements are taken every 10 minutes. Measurements during the person's activity is much noisier, which requires the use of appropriate mechanisms for signal processing. For the improvement of the processed signal there was used wavelet analysis method. There were tested a number of photonic sensors, FBG type: refractometers, hygrometers, the presence of EM field, magnetometers, as well as multi-sensors immunized for a specific interfering parameter.

There is continued to be developed in ISE PW a medical device for TEWL (trans epidermic water loss) parameter measurements. It bases on dew point detection and uses a capacitive sensor. It measures water loss through the skin in a standardized manner and presents the measurements in a form of a single objective parameter. There were fabricated several models with different structures of the measuring chamber, open, closed and with a cold trap. The aim is to improve the measurement accuracy, linearity, frequency, and optimizing the working conditions of the semiconductor detector.

A team at Lublin University of Technology is building the test, laboratory, distributed sensor network system for monitoring, potentially, the municipal heating network. The purpose is the economization of practically operated system in which there is observed a significant loss of heat due to many heterogeneous reasons. As the sensors there are used thermoelectric Peltier units. The cell is constructed of two semiconductors having different values of Seebeck coefficient. In the laboratory there was constructed a measurement system appropriate to the operating conditions. The aim was to show the usefulness of the system for detecting the transit of heat in the pipes of the heating network of the city.

On the West Pomeranian University of Technology in the laboratory of photonics there is constructed an interferometric measuring system for chromatic dispersion of microstructured optical fibers. The work is conducted in collaboration with ITME, UW and InPhoTech. Microstructured fibers are used for the construction of photonic sensors. The aim of the study is to review the practical usefulness of interferometric methods to measure chromatic dispersion, which is the sum of material and waveguide dispersion, and construction of a useful set for research. The resulting accuracy of the measurements in the laboratory conditions were a few\%.

The ISE PW, in cooperation with IFPiLM is doing research on optimizing the working conditions, reading and processing of data from the GEM detectors. Multilayer GEM sensors are complicated, and the output includes a multitude of data which must be "decoded" before they are useful for the analysis. The sensor area includes several sub-areas, like drift, transfer, avalanching and collection of charge. The aim is to minimize the sensor latency and maximize the data rate. System optimization is to introduce additional feedback operating in the real time. Greater bandwidth and reduced latency has been obtained by parallelization of data processing.

Modern, miniature sensors find numerous applications in systems of technical diagnostics, telemetry, built-in monitoring, protection, alarms, safety, status, etc. Research on sensor-equipped system fault tolerance (software and firmware layers) is one of the current topics of research conducted in the II PW. It is necessary to create a relevant testing environment of tracking changes after injection errors, simple or more complex ones. Embedded, microcontroller based diagnostic, multi-channel system is built with limited resources and access. Telemetry tracking terminal (TTS) communicates with the group of peripheral devices, which are various, and numerable sensors. The data is transmitted to the central unit wirelessly and is used for monitoring the device under test. The system under control may be a vehicle, autonomous functional machine, a drone, a robot, etc. The gathered data 
are collected by a central station and serve for failure prediction, estimation of breakdown costs, estimation of control intercept danger, etc. TTS supports alarms and additional security related to operational safety. There is also evaluated a dependability of the TTS system. Requirements for the dependability of the diagnostic and monitoring sub-systems were classified into three groups, in terms of impact on the functionality of the monitored system - primary, secondary and other/auxiliary. Primary requirements determine the fundamental principles of operation of the system. Secondary requirements determine quality of work. The TTS equipment consists of many peripherals and sensors (accelerometers, gyroscopes, GSM, GPS, system location, physical size, power consumption, etc.), and analogue I/O, digital buses and communication links (I2C, SPI, UART, ADC, etc.). All components are connected to the central unit, which is a microcontroller. TTS can be modified by software bootloader residing in a flash. Separately from this, one can modify the firmware based on FreeRTOS operating system (OS). Time and position stamped, aggregated data are stored on the SD card. Dependability of the TTS was tested by injecting errors in different places of the operating unit including a microcontroller, OS and tested for the operation and stability. The study uses the JTAG interface. TTS device is developed to extend the monitoring capabilities of more complex systems. Dependability analysis was performed on other devices like network routers, using standardized errors injection technique. Usually, the standard firmware exhibits low immunity to the external interference.

Distribution of reference time and frequency is a very important issue (theoretically and technically) in many applications, such as extremely precise synchronization of events and processes, precise location, mapping of events in time and space, navigation, research, etc. In the high quality distributed networks of sensor there are used accurate methods for the transfer of time and frequency. The network compares the reference clocks to produce the time scale, for example the UTC. The examples include such systems like GNSS TWSTFT, PTP, NTP, FOL, etc. Work on ultra-precision fibre optic systems for the transfer of time are conducted at AGH. The tests use fibres leased from telecom operators. Time comparison of distant clocks, and behind them the status of the sensors, is done by signal transmission with feedback using precise bi-directional links. Along the long link the optical signal is amplified by EDFA components. Practical distribution network of time standard is multi-accessible and distributed. Jitter is accumulated in the network, and has to be measured in numerable points. There has been developed a concept of measuring the signal distortion using PLL based fast broadband feedback system tracking the jitter, and narrowband feedback to remove the jitter. Laboratory tests were carried out on several sections of the multi-hundred-km fibre lengths introducing signal distortion to simulate various levels of the jitter. Based on the simulation and measurement results, the efficiency was optimized of a standard single mode optical fibre link. The test also included the research on the influence of the Brillouin backscattering to the reference link efficiency and precision.

There is under development a system that integrates video signals from a large number of various sensors and heterogeneous sources. Work is carried out under the project
ISW (Integrated Video System) in cooperation of ISE PW, WSPol, Atende Software and Vortex. Requirements for the data acquisition system are the following: automatic acquisition of various video signals, encoding and decoding, transmission over Ethernet, scalability, global extent in the whole country, full backward compatibility, the interfaces to the existing and currently operated systems, three levels of access - administrator, active users and passive user, long-term support and the ability to upgrade. The hardware module has HDMI, HD-DVI, CVBS inputs and the Ethernet. The equipment includes SoC - FPGA and ARM, H.264/256, motherboard PCB and 2 FMC cards, packaged in a simple MTCA case. The software is modular, supports the ONVIF and RTSP, has access to the database, terminal management by JSON, and is location aware. Implementation of the hardware and software, after appropriate testing, is expected within one year.

To protect the network systems, including network sensor systems, there are used IDS/IPS class (intrusion detection intrusion prevention) methods. The development and research on such cybersecurity systems, based on the transmitted data (not additional external data), are carried out in ITele PW. These systems operate in different modes of work. Detection of attacks carried out in the signature based system is based on signatures of known descriptions and stored in the database. Detection of attacks in the anomaly analysis system is based on the decision of whether the activity belongs to a group of normal, nominal, or not. The "nominal-anomalous" (NA) systems are monitoring all the time protocols, packets and data flows. Experiments were conducted with combining the idea of building the IDS/IPS module with the classical methods of machine learning using artificial neural networks with a multilayer perceptron. A method of back-propagation and optimization of a swarm of particles in order to search for the best algorithm for classifying the flow of network data was tested. The system was checked on standardized and corrupted data. There was developed a model based on the following parameters: packets flow, octets flow, duration, source port, destination port, TCP flags, XORed, and the IP protocol.

Cybersecurity systems for computer networks are also built using the technique of Trust and Reputation (TRS) - a trusted reference system. The ITele PW cybersecurity lab is working on the reliability of the TRS. Conventional methods of security based on cryptography are often insufficient. The aim of the TRS is to identify network nodes that act selfishly, that is, pursue their goals and not to communicate which is a fundamental objective of the network, act maliciously or disrupt network communications. It is of course the simplest case for detection. Intelligent intrusion methods do not work this way. The way to prevent such simple behaviour is to integrate and consolidate the knowledge of all network nodes. Each node observes and is aware of the behaviour of the neighbours. The nodes exchange opinions and recommendations of other nodes. This prevents the selfish behaviour of infected nodes, but does not prevent the attack on the trust TRS system itself. The research issue is the safety of widely used systems of trust, which are commonly applied in networks like WSN, MANET, P2P platforms, e-commerce, social networks, etc. The advantage of application of trust servers is a partial strengthening of security. Disadvantages are: increased system complexity, increase in the required 
transmission bandwidth, additional resource requirements for memory and processing power, and above all a considerable susceptibility to direct attacks on the trust servers. The currently conducted research focuses on performance metrics of trust and reputation servers and to compare such measures in case of exposure to standardized simple attacks, tactical and strategic, and collaborative attacks. The attacks may, for example, especially insidiously lead to a reduction in confidence, in not properly/sufficiently defended real trust server. Research methodology includes cataloguing the attacks, creating a network model and the model of vulnerability, analyses the characteristics of the network and its parameters, and test scenarios, including the optimization of the behaviour of the attacker.

\section{BIOMEDICAL ENGINEERING, BIOPHOTONICS, OPTOGENETICS}

Biophotonics is developed in Poland in cooperation between biological, biomedical, and technical institutions active in the field of biology, biomedical engineering and photonics. Such a collaboration, between Nencki Institute, CFT PAN and ISE PW, gives interesting results of this unique research. This development is also reflected in Wilga in the form of thematic sessions. Biophotonics already developed more intensively for more than two decades combines seemingly distant fields of science and technology such as medicine and biology, physiology and photonics. It attracts the interest of young scientists. Biophotonics uses traditional research tools like optical microscopy and creates new modifications of these tools as multi-spectral imaging techniques, sheets of light, super-resolution imaging, modified confocal microscopy, fluorescence microscopy, Fourier microscopy, hybrid imaging techniques, fluorescence microscopy, total internal reflection or TIRFM technique, and many others. There are developed specific methods of induction of optical signals, as research tools, within the respective cells, especially in neural cells of living organisms, and finer detection methods of such biooptical signals. Biophotonics also gives impetus to the development of specialized tomographic techniques, in addition to those existing and used for general purposes. The techniques of classical optical computer tomography (OCT) are subject to differentiation, for example, in the direction of optical diffusion imaging DOI, diffuse optical tomography DOT, and others. Biophotonics uses strictly selective active methods of interaction with the tissues and living organisms in order to induce the desired response requested and visualizing the researched physiological processes and to emphasize their details, including succession of the biological chain reactions, some of which have mechanisms related to photonics and energy conversion. Many of these new biophotonic tools are able to reveal subtle cellular reactions, collective tissue reactions or a more complex response of the body parts or even the whole body. Fluorescent resonance energy transfer spectroscopy FRETS, combined with specialized microscopy techniques, in biophotonic research uses two fluorophores applied in the biological environment. The energy is transferred between the fluorophores mutually, nonradiatively, to the right biological location. The local neurons are activated or de-activated genetically to sensitize them to particular photon energy. Using opto-genetics techniques on the neurons there are examined deep internal mechanisms underlying the macro-behavioral phenomena.

Biophotonics uses much more brand new photonic tools that have been developed only with a significant increase in interest in this field, especially in recent years. There is developed a field of dynamic functional imaging, the potential of which is not yet fully exploited because of the need for adaptation and acceptance, as well as standardization of the numerable methods for biomedical conditions. The developed research methods relate, for example to selective laser microstimulation of tissues, cells, inside-cell organs, stimulation on single-photon level of organelles and molecular structures, etc. Photonics has the tools to build optical nano-resolution tweezers operating in the in-vivo environment. Optical fiber catheters with a diameter of tens of micrometers can be used to transfer and observe energy conversion inside the tissue, to initiate or localize the investigated reactions. Such fiber optic version of nano-capillary deliver/receive to/from the reaction sites selective energy and biochemical materials, in a multichannel way. One can also mention the tissue and cell-level nano-energizers, nano-coagulators, scalpels and photonic nano-manipulators. The Wilga sessions on biophotonics debated some of the issues discussed above, and also the construction of advanced photonic instrumentation for biomedical research. The sessions showed a considerable progress in that work as compared to the last year and previous years. Biophotonics sessions are systematically organized in Wilga for several years.

Biophotonics contains a very fast growing sector - which is optogenetics. The work in this area is conducted in Poland in several centers, for example, Nencki Institute for Experimental Biology of PAS in cooperation with CFT PAN, and ISE PW. Optogenetics concerns a study of physiological and behavioral phenomena at different functional level in living organisms modified by the sensitization of the local neuronal centers to the impact of light. Optogenetics raises hopes for detailed progress in the field of brain research and the operation of particular parts of the nervous system. Generally, it involves photon interaction, instead of electron and ion on the special places in the body at the neural level, also sometimes subcellular. Here we are interested in animal behavioural changes when exposed to light acting directly on selected neuronal structure. Optogenetics session was organized in Wilga in the years 2014, 2015, and was now continued in 2016. Optogenetics research on mice is conducted at the Nencki Institute including multiple aspects of the behavioural changes of the animal including the conditioned reflexes, unconditional reflexes, dependence on the type of food, alcohol, social habits, the forces of habits, etc. Several years of the research project led to the creation of a unique interdisciplinary team and developed specific tools and research methods. To study the animals there were used modified advanced living environment of the IntelliCage class. The modifications included the improvement of measurement systems, like very exact location of all animals, even in the event of a significant grouping. During the project there were designed and manufactured hardware and programming tools consisting of an implanted part and outside complex measuring system coupled with the intelligent living space. The implanted part is inductively powered, and its structure is as follows: the coil and the receiver clock, analogue multichannel input stage for 
the measurement of biological signals, multi-axis accelerometer, wi-fi receiver adapter, microcontroller, power management, battery, DEL drivers, etc. The implanted part was highly optimized for the use of power. During the Wilga optogenetic session, there was presented the progress of biological research using photonic equipment.

Cerebral neurons in mice and rats, on which the optogenetic research is conducted, typically have dimensions of $30-50$ microns and they are densely packed with intervals between them of the order of several to 100 microns. How can one precisely control so small and densely packed cells? There are significant difficulties in the selective stimulation of one cell types without affecting the other. Even more difficult to selectively stimulate there are selected neural signal paths, because some of them run on the complicated way, linking completely different brain structures, and the length of this path can be up to several $\mathrm{cm}$. The idea of the optogenetics is to exert selective effect on selected groups of neurons and signaling pathways. Light-sensitive proteins function as ion channels in cells and may affect their functions. Halorhodopsin is a cellular and synaptic inhibitor $(\mathrm{Cl}-, 580 \mathrm{~nm}$, yellow light). Channel rhodopsin activates cells and synapses ( $\mathrm{Na}+, 470 \mathrm{~nm}$, blue light). These materials, and light must be supplied separately to the respective groups of neurons of a living animal. The protein is delivered to the relevant place by viruses. The viruses are injected in appropriate locations in the brain. Channel rhodopsin is embedded in the synthetic DNA of the virus. The beam of light through miniature fibre optic catheter is activating channel rhodopsin around the studied place in the brain. Other developed solutions is based on the sub-miniature cannula ended with a miniature light emitting diode covered by a thin layer of efficient type of quantum dots phosphor. The intensity of radiation in the cannulas tested in mice was in the range of $15-20 \mathrm{~mW} / \mathrm{mm}^{2}$. The cannulas were initially tested in vitro and are prepared for testing in vivo. The test environment and test results are examined from the point of view of biodiversity.

One of the goals of the research is to study the mechanisms of fear and phobias, which are common psychological phenomena and play a significant role in social interactions. Which structures of the brain are responsible for these phenomena. Optogenetics studied the potential neuronal connections paths between distant brain structures (hippocampus, amygdala, prefrontal cortex) responsible for fear responses, subjecting the animals by cyclical expositions and recovering or erasing optogenetically the memory of fear. There was studied the transfer of social fear, through effects on the central part of the amygdala. The started successful optogenetic research indicates its suitability to explore particular roles of specific neuronal circuits in the brain. The advantage is the ability to study optogenetically excited social interactions in freely moving animals. In technical terms, work is underway on further miniaturization of the optogenetic equipment and transition to a generation of completely wireless. Further research plans relate to the development of research with optogenetic animals measured and accurately localizable in wireless environment of the IntelliCage. For such a research environment there is created a next-generation with implanted microprocessor and micro-peripherals, and the rest of advanced measuring equipment on the $\mathrm{PC}$ side, and full software for the entire system. IntelliCage environmental is added with full wireless multichannel measurements function including the exact location of all animals. The requirements for the wireless optogenetic stimulating and measuring system are as follows: dimensions resolution of a single $\mathrm{mm}$, multiple control channels for LED, multi-channel data acquisition ECG and EEG electrodes, wireless data, long-term battery -order of many weeks, local accurate positioning, system device implantable under the skin without disrupting social behaviour of the animal, low cost, and effective cooperation with the environment IntelliCage.

In biomedical research, the absolutely fundamental principle is repeatability. Planning an experiment in which such a rule is violated is not allowed. The source of uniqueness and nonrepeatability are custom, unclear experimental assemblies, custom data analysis, bad use of statistical tools and publishing errors. The solution to part of the problems is the automation of measurement systems which minimizes the impact of the experimenter, formalization of decision-making criteria, a significant accuracy of observation and standardizing the conditions of the experiment. The standardized measurement environment, the IntelliCage was used in the research conducted by ontogenetic team from IBD PAN. IntelliCage allows to conduct long-term experiments, examining social context, reducing the stress for tested the animals, and flexible configuration of the experimental situation. In cooperation with CFT PAN and ISE PW there are carried out works on additional instrumentation for the IntelliCage test environment for the much needed functionality, such as exact location and orientation of the individual animals in a larger group. IntelliCage has a number of built-in functionalities, including ones of interactive nature, in the standard instrumentation. The full system is a generator of large amounts of data, correlated in various complicated ways. A non-trivial question arises about the reproducibility of the experiment in such difficult conditions, a significant number of methods, parameters, statistics error, animal behavioural issues, etc. The solution seems to standardize further the study of large sets of data for the IntelliCage environment. The study used a dedicated environment IC library Python PyMICE.

AGH team is building a modular sophisticated system of electrical stimulation of the brain of a moving animal, and multichannel recording of the animal activity. Neurostim software was used to record neurobiological signals commands, stimulus generation, etc. Developed measurement equipment consisting of a miniature direct interface card, biological communication interface and PC interface. There was developed data acquisition software and virtual system operator. The system is tested in laboratory and soon will provide tests with animals.

Biomedical part of Wilga Symposium has extended in recent years and now includes several topical sessions. The session on biomedical engineering was organized by prof.A.Grzanka from ISE PW. Several articles related to theoretical and experimental analysis of human gait were presented. The aim of the work carried out in parallel by several research teams is to help people with disabilities to regain mobility, and the rehabilitation optimization issues of such persons. Other objectives of this study are related to cooperation with schools and sports athletes in order to optimize the training effort. Another area of cooperation concerns the research programs on exo-skeletons structures for 
people. A group of research work was related to the research of genetic code of certain plants, especially cucumbers. Cucumber genome research is a Polish specialty in the international scale. Group of articles were related to bioinformatics systems based on genetic information, and other bio-inspired information.

A separate session on biomedical engineering showed the progress of work on large construction project of an on-line system for the detection of cancers. One of these projects is related to a proprietary solutions of dedicated sensor for breast cancers based on the liquid crystal surface sensors. The sensor is present on-line and makes the breast image acquisition, image uploading automatically over the Internet to an external data processing system for the evaluation and diagnosis. The system is powered by a large database of similar/relevant images. The project is commercial in nature, it is funded by the Braster and relates to the comprehensive solution of the hardware and software. The result will be a popular inexpensive sensor for purchase at the pharmacy together with the time subscription to use. The session was organized by $\mathrm{dr}$. hab. R.Nowak from ISE PW, and was based on biologically inspired computing systems and the processing of visual information. Another example of advanced image processing was a system for gas leaks detection used in the industry.

EMG signal processing in the real time is used in the construction of bionic prosthetic limbs. The works are conducted in ISE PW. Readings of physiological bioelectric signals from the muscles of a limb or nerve signals are used to control the prosthesis. The next step is to read the control signals from the brain. The signals obtained directly from the nerves require invasive implants. Readouts of the EMG signals require only external electrodes. Preliminary measurements were directed toward the distinguishability of the format of the signals collected from the skin at different locations during the selected movements of forearms. An algorithm for operation of the measuring system was written. There were optimized signal processing conditions including their filtering, noise reduction, becoming independent from the type of equipment and the recording method, windowing, conditioning and digital conversion.

\section{WILGA 2016, WILGA 2017}

Optics and photonics, electronics, and sensors, are developing very intensively in the fields of research and applications. One can distinguish the development on the global and local levels. They slightly differ from each other. Big projects related directly and indirectly to the optics, photonics and similar areas generally require the involvement of large laboratories and certainly of great resources. These big projects are combined with large laser and synchrotron infrastructures, and expensive technology lines. Large-scale technological infrastructures concern wide research on semiconductors and dielectrics, and other materials for photonics, manufacturing of optical fibres, planar integrated optics, photonic sensors, etc. Local research undertakings concern rather significantly smaller initiatives, often university based, also conducted at departmental institutes. Some of the most important local initiatives also have an international character. The papers of young scientists presented during the Symposium WILGA 2016 reflect the state of optics and photonics and advanced electronics in Poland, and also in some European laboratories. This image is not too bad.

The next Symposium 2017 WILGA Applications of Photonics and the Internet Engineering will also be organized in two editions the 39th and 40 ${ }^{\text {th }}$, Winter 27-29 January 2017 and Summer 28 May - 05 June 2017. The organizers of the Symposium WILGA 2017 invite young scholars and their mentors to participate [1-2].

\section{REFERENCES}

[1] Sympozjum WILGA: http://wilga.ise.pw.edu.pl

[2] Sympozjum WILGA: https://pl.wikipedia.org/wiki/Sympozjum_Wilga

[3] J.W.Modelski, R.S.Romaniuk, Committee of Electronics and Telecommunications, Polish Academy of Sciences, Structure Activities - Perspectives, International Journal of Electronics and Telecommunications, 2015, vol.61, no.1, pp. 49-56, doi:10.1515/eletel-2015-0007

[4] R.S.Romaniuk, WILGA Symposium on Photonics Applications, Photonics Letters of Poland, 2009, vol.1, no.2, pp.46-48, http://photonics.pl/PLP/index.php/letters/article/view/1-16

[5] R.S.Romaniuk, J.Dorosz, W.Wójcik, P.Mergo, R.Buczyński, Optical fiber technology in Poland - four decades of development 1975-2015, Proc.SPIE 9816, 2015, doi: 10.1117/12.2197804

[6] R.S.Romaniuk, Photonics applications in astronomy, communications, industry, and high-energy physics experiments 2015, Proc.SPIE 9662, 2015, doi: 10.1117/12.2068107

[7] R.S.Romaniuk, Photonics applications in astronomy, communications, industry, and high-energy physics experiments 2014, Proc.SPIE 9662, 2015, doi: 10.1117/12.2068107

[8] R.S.Romaniuk, Electronic and Photonic Systems WILGA 2014, International Journal of Electronics and Telecommunications, 2014, vol.60, no.3, pp.271-276, doi:10.2478/eletel-2014-0035

[9] R.S.Romaniuk, Photonics applications in astronomy, communications, industry, and high energy physics experiments, Proc.SPIE 8903, 2013, doi:10.1117/12.2035420

[10] R.S.Romaniuk, K.T.Poźniak, Photonics applications in astronomy, communications, industry, and high Energy physics experiments, Proc. SPIE 5125, 2003, doi:10.1117/12.531519

[11] R.S.Romaniuk, Zaawansowane systemy elektroniczne - Wilga zima 2016, Elektronika - konstrukcje, technologie, zastosowania, vol.57, nr.5, str.35-39, 2016, doi:10.15199/13.2016.5.7

[12] R.S.Romaniuk, Zaawansowane systemy fotoniczne i elektroniczne oraz inżynieria Internetu - Wilga 2015, Elektronika - konstrukcje, technologie, zastosowania, vol.56, nr.11, str.116-123, 2015, doi:10.15199/13.2015.11.29

[13] R.S.Romaniuk, Zaawansowane systemy elektroniczne i fotoniczne, część 4 - nanomateriały I systemy elektroniczne dla techniki kosmicznej, Elektronika - konstrukcje, technologie, zastosowania, vol.56, nr.2, str.93-100, 2015, doi:10.15199/13.2015.2.22

[14] R.S.Romaniuk, Zaawansowane systemy elektroniczne i fotoniczne - Wilga 2014, część 3 - Elektronika biomedyczna i multimedia, Elektronika - konstrukcje, technologie, zastosowania, vol.56, nr.1, str.48-55, 2015, doi:10.15199/13.2015.1.9

[15] R.S.Romaniuk, Zaawansowane systemy elektroniczne i fotoniczne - Wilga 2014, część 2 - Systemy elektroniczne dla eksperymentów fizyki wielkich energii, Elektronika konstrukcje, technologie, zastosowania, vol.55, nr.12, str.41-49, 2014, doi:10.15199/ELE-2014-219 
[16] R.S.Romaniuk, Zaawansowane systemy elektroniczne i fotoniczne - Wilga 2014, część 1 - Fotonika, Elektronika konstrukcje, technologie, zastosowania, vol.55, nr.11, str.117127, 2014, doi: 10.15199/ELE-2014-208

[17] R.S.Romaniuk, Zastosowania fotoniki i inżynieria Internetu, XXXVIII Sympozjum WILGA, Elektronika - konstrukcje, technologie, zastosowania, vol.57, nr.8, 2016, w druku
[18] R.S.Romaniuk, Zastosowania fotoniki i inżynieria Internetu, XXXVIII Sympozjum WILGA, Elektronika - konstrukcje, technologie, zastosowania, część 2, vol.57, nr.9, 2016, w druku 